\title{
The feasibility of using ultrasound during follow-up for superficial non-melanoma skin cancers after electronic brachytherapy
}

\author{
Uma Goyal, MD, Justin Suszko, MD, Baldassarre Stea, MD, PhD \\ Department of Radiation Oncology, University of Arizona, Tucson, Arizona, USA
}

\begin{abstract}
Purpose: Non-melanoma skin cancers (NMSCs) can be treated with a number of modalities including surgery, topical chemotherapy, or radiotherapy. Amongst the radiotherapeutic options, electronic brachytherapy (eBT) is an appealing treatment as it is usually given in a few fractions, it leads to good outcomes, and is increasingly being used. However, currently no follow-up imaging is routinely used or recommended to evaluate treatment response of NMSC. We aimed to use ultrasound (US) in follow-up after eBT for superficial NMSC to assess its feasibility in detecting possible tumor response.

Material and methods: Fourteen patients were treated between 2013-2015 for a NMSC using eBT. US guidance was used for treatment planning prior to eBT initiation. After completion of eBT, patients were seen in follow-up for both clinical exam and a repeat US at 1 month to evaluate if tumor response was detectable.

Results: Of the 14 patients, 6 were male and 8 were female. The mean age was 71 years. With a median follow-up of 20.5 months, all patients had a complete response based on physical exam. Eleven patients appeared to have a complete response based on US obtained $>1$ month after completing eBT. To date, there have been no local recurrences or progression, and all patients are alive.

Conclusions: US is an objective imaging modality that may be able to assess NMSC response after eBT. Based on follow-up imaging, further treatment or observation may be recommended. Although this study is hypothesis generating, larger studies with pathologic confirmation of recurrences would be needed to validate US use for follow-up, avoiding possible painful and scarring biopsies in case of low suspicion of recurrence.
\end{abstract}

J Contemp Brachytherapy 2017; 9, 6: 535-539 DOI: https://doi.org/10.5114/jcb.2017.72358

Key words: brachytherapy, electronic, skin cancer, ultrasound.

\section{Purpose}

Non-melanoma skin cancer (NMSC) is the most common cancer in the United States, with an incidence greater than that of all other cancers combined [1]. It has been reported that basal cell carcinoma (BCC) has 2-5 times the incidence than squamous cell carcinoma (SCC) of the skin. Superficial NMSCs usually have a good prognosis with treatments ranging from topical chemotherapy, surgery, and radiotherapy $[2,3,4,5,6,7,8,9,10,11,12]$. Despite having treatment guidelines for NMSCs that provide a decision making process at most steps from diagnosis to follow-up, observation remains as the follow-up recommendation after treatment $[1,2,13,14]$. However, there has been a suggestion that follow-up guidelines for treated NMSCs may not be clear [15].

The overall recurrence for nodular, superficial, and sclerosing subtypes of BCC at 5 years was found to be about $16 \%$, and for cutaneous SCC was approximately $5 \%$ at
10 years [16,17]. Although this is a small risk, there are certain factors of SCCs found to have poor outcomes such as diameter $>2 \mathrm{~cm}$, invasion beyond fat, poor differentiation, perineural invasion, and location on ear, temple, or anogenital area. Cutaneous SCCs can also lead to metastases $4 \%$ of the time, and have a $2 \%$ disease-specific death $[4,17]$.

In order to evaluate treatment response and monitor treated NMSCs for possible tumor recurrence, we studied the feasibility of ultrasound (US) as an objective imaging modality during follow-up after electronic brachytherapy (eBT) completion. To our knowledge, there are no other studies showing the use of US in follow-up after treatment for NMSC, particularly using eBT.

\section{Material and methods}

From December 2013 to June 2015, 15 patients with 15 biopsy-proven NMSCs were treated with definitive eBT (Axxent eBx; Xoft - a subsidiary of iCAD, Inc., Sunnyvale, 
CA, USA) using US guidance for treatment planning. A pre-treatment US was done to determine depth and diameter of the NMSC, based on previously published methods [18]. The pre-treatment US depth was used for dose prescription. Eligible patients for this feasibility study had both a pre-treatment US for eBT planning and post-treatment US completed at least 1 month after eBT completion for a superficial NMSC of $<5 \mathrm{~mm}$ depth. One patient was ineligible due to having a post-treatment US done at fraction 8 of 10 of eBT, instead of at least 1 month after completion of eBT. There were 14 eligible NMSCs in 14 patients for this retrospective feasibility study.

Radiation oncology and radiology, referring physician documentation, and hospital records were reviewed. Side effects were scored according to the National Cancer Institute Common Toxicity Criteria for Adverse Events, version 4 . This study was approved by our institutional review board. Written consent from each patient was obtained to publish data and photographs.

\section{Treatment details}

Patients were treated using eBT at a dose of $40 \mathrm{~Gy}$. Fractionation schedule consisted of 10 fractions, 4 Gy each, and lesions were treated 2-3 times a week. No extra margin was added to depth based on US measurements

Table 1. Patient and lesion characteristics

\begin{tabular}{|c|c|c|}
\hline & \# Patients & $\%$ Patients \\
\hline \multicolumn{3}{|c|}{ Age (years) } \\
\hline $40-65$ & 5 & 36 \\
\hline$>65$ & 9 & 64 \\
\hline \multicolumn{3}{|l|}{ Gender } \\
\hline Male & 6 & 43 \\
\hline \multirow[t]{2}{*}{ Female } & 8 & 57 \\
\hline & \# Lesions & $\%$ Lesions \\
\hline \multicolumn{3}{|l|}{ Histology } \\
\hline $\mathrm{BCC}$ & 9 & 64 \\
\hline SCC & 5 & 36 \\
\hline \multicolumn{3}{|c|}{ Pre-treatment depth (mm) } \\
\hline $1-1.9$ & 6 & 43 \\
\hline $2-2.9$ & 5 & 36 \\
\hline $3-3.9$ & 2 & 14 \\
\hline $4-4.9$ & 1 & 7 \\
\hline$\geq 5$ & 0 & 0 \\
\hline \multicolumn{3}{|c|}{ Pre-treatment largest cross-sectional diameter (mm) } \\
\hline $1-5$ & 5 & 36 \\
\hline $6-10$ & 7 & 50 \\
\hline $11-15$ & 1 & 7 \\
\hline $16-20$ & 1 & 7 \\
\hline
\end{tabular}

$B C C$ - basal cell carcinoma, SCC - squamous cell carcinoma and Mohs pathology data showing that US may overcompensate for depth by about $27 \%$ [19], and there was no expected setup error in the vertical dimension.

\section{Ultrasound measurements}

Focused US of the lesions was performed by a board-certified radiologist using either: the LOGIQ E9 (GE Healthcare, Milwaukee, WI, USA), or S-3000 US units (Acuson; Siemens Medical Solutions, Mountain View, CA, USA). Linear array transducers with frequencies of 14 and $18 \mathrm{MHz}$ were used to obtain images. Imaging was performed with either a custom made gel standoff pad or cold US gel acting as a standoff, especially on body parts where the gel pad could cause image degradation. Gentle scanning was done to avoid distortion of NMSCs. Two-dimensional B-mode images were obtained with transverse and longitudinal sweeps across the lesion. The US probe was oriented along the longest dimension of the lesion to obtain sagittal and transverse evaluations. Measurements of depth were made at the most vertical extension of the NMSC in either plane [18].

US images were obtained pre-treatment for eBT planning purposes, and were again obtained $>1$ month after completing eBT during follow-up (at a median follow-up of 6 months). The exact timing of follow-up US was at the discretion of the treating radiation oncologist. The pre-treatment and post-treatment US images were compared for complete response (CR), partial response (PR), or no response (NR). CR was defined as no residual tumor seen on post-treatment US. PR was defined as any decrease in cross dimension measurements on post-treatment US. NR included lesions of the same size or larger on post-treatment US. Residual disease seen on US was defined as a well-defined echogenic discrete circumscribed lesion, rather than heterogeneity of echogenicity commonly seen post-eBT on US from inflammation. Feasibility was defined as the ability to visualize $C R$ versus PR versus NR on post-treatment US, based on a board certified radiologist's interpretation.

\section{Results \\ Patient and lesion characteristics}

There were 14 eligible NMSCs in 14 patients. Mean age of patients was 71 years, with 6 males and 8 females. There were 9 BCCs and 5 SCCs, with 4 nodular BCCs, 3 superficial BCCs, 1 superficial-nodular BCC, 1 well-differentiated SCC, 1 moderately-differentiated SCC, 1 well to moderately-differentiated SCC, and 3 lesions were not specified with subtype. The pre-treatment largest diameter of treated NMSCs on US ranged from $4 \mathrm{~mm}$ to $17 \mathrm{~mm}$. The mean pre-treatment depth of treated NMSCs on US was $2.1 \mathrm{~mm}$ (1.3-4 mm). After completion of eBT, no lesions were visualized or clinically palpable at subsequent routine follow-up with a radiation oncologist. Table 1 summarizes patient and lesion characteristics.

\section{Ultrasound of NMSCS}

Eleven post-treatment US completed at a median of 6 months post-treatment showed no residual disease 
(CR) after eBT. Six were BCC and five were SCC. Their initial measurements were a mean depth of $2.0 \mathrm{~mm}$, and the largest diameter was $17 \mathrm{~mm}$.

Three post-treatment US completed at median of 6 months after eBT showed residual disease (PR). All 3 were BCC, one of which was recurrent BCC after Mohs micrographic surgery. Their immediate post-treatment clinical exam showed erythema in all 3 PR patients with eschars in 2 patients. Their initial US measurements were a mean depth of $2.2 \mathrm{~mm}(3 \mathrm{~mm}, 1.5 \mathrm{~mm}, 2 \mathrm{~mm})$, and a mean of largest diameter was $7.7 \mathrm{~mm}(7 \mathrm{~mm}, 12 \mathrm{~mm}$, $4 \mathrm{~mm})$. Their initial clinical exam measurements were a mean depth of $2 \mathrm{~mm}(3 \mathrm{~mm}, 1 \mathrm{~mm}, 2 \mathrm{~mm})$, and a mean of largest diameter was $10 \mathrm{~mm}(7 \mathrm{~mm}, 20 \mathrm{~mm}, 3 \mathrm{~mm})$. Their post-treatment US measurements showed a mean depth of $1.7 \mathrm{~mm}(2 \mathrm{~mm}, 1 \mathrm{~mm}, 2 \mathrm{~mm})$. The third PR patient's post-treatment US report described a less conspicuous lesion with post-radiation changes compared to the pre-treatment US.

None of the patients with residual disease on follow-up US had progression of disease (NR). Two post-treatment
US reports described post-radiation changes. However, neither crusty lesions, bleeding, nor cutaneous infections were described as problems for post-treatment US visualization. Figure 1 shows pre-treatment and post-treatment US images for a NMSC with a CR, and another NMSC with a PR.

\section{Treatment outcomes}

All patients have had a clinical CR-based on physical exam (inspection and palpation); however, $78.6 \%$ of patients had a radiologic CR-based on follow-up US imaging. The $21.4 \%$ of patients with a radiologic PR were monitored with more frequent follow-ups, and clinical exams with radiation oncologists and dermatologists. The first patient with PR had a post-treatment US done at 6 months, and was seen again 5 months after US showing no clinical evidence of residual disease. Slight hypopigmentation at the treated site was noted and the patient was referred to dermatologists for clinical exams. The second patient with PR had a post-treatment US done at 7 months, and was seen every 1-2 months for 4 months
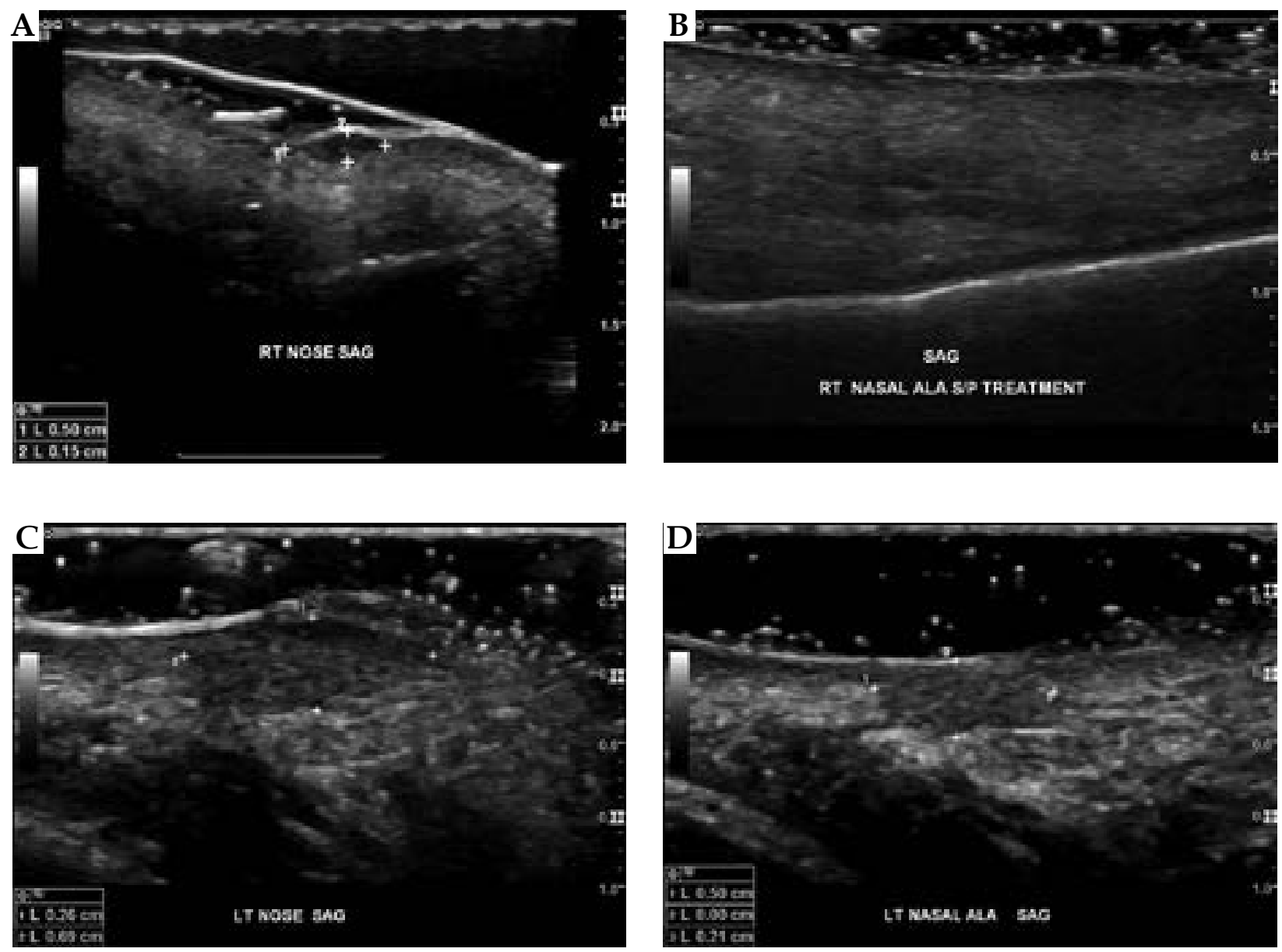

Fig. 1. Pre- and post-treatment ultrasound imaging. A) Pre-treatment ultrasound imaging shows the depth and diameter of a hypoechoic right nasal ala non-melanoma skin cancers (white crosses). B) Post-treatment ultrasound imaging shows no evidence of residual tumor along the right nasal ala. C) Pre-treatment ultrasound imaging shows a hypoechoic lesion on the left nasal ala measuring $0.69 \mathrm{~cm}$ in diameter and $0.26 \mathrm{~cm}$ in depth (white crosses). D) Post-treatment ultrasound 6 months later demonstrates interval reduction in size of the same lesion, now measuring $0.50 \mathrm{~cm}$ in diameter and $0.21 \mathrm{~cm}$ in depth consistent with a partial response 
for clinical exam with radiation oncologists showing no evidence of residual disease but mild hypopigmentation, and then referred to dermatologists for routine skin exams. The third PR patient had post-treatment US at 2 months and was seen at the completion of treatment by radiation oncologists, and found to have moderate erythema. The attending radiation oncologists called the patient 1 month post-treatment due to patient's inability to come to clinic due to personal reasons, and the patient followed post-treatment with dermatology clinical exams with no evidence of disease recurrence.

With a median follow-up of 20.5 months (range, 8.540 months), there have been no progression noted on clinical exams. Also, there were 7 cases of grade 1 hypopigmentation, 2 cases of grade 1 erythema, 1 case of grade 2 erythema, and no cases of grade 3-4 skin necrosis or delayed healing after eBT. All patients are alive.

\section{Discussion}

This study reports on the feasibility of using standard US technology as a follow-up tool after eBT for NMSCs, to determine treatment response of a lesion after eBT. The information from these images may have the potential to be used to determine possible need for salvage treatment. Salvage treatments of recurrent NMSCs include surgery and/or additional radiotherapy [20,21,22,23,24]. The mean time to $\mathrm{BCC}$ recurrence has been reported to be 20 months, with the majority of recurrences occurring within 3 years [16], and it has been reported that at 10 years, cutaneous SCC recurrence is about 5\% [17]. Risk factors for recurrence of NMSCs include a history of multiple skin cancers, immunosuppression, and pathologic features of the NMSC $[17,24,25]$.

In a published work, the $18 \mathrm{MHz}$ US depth was compared with punch biopsy to determine the depth of lesions. The results showed significant discrepancies in depth determination: US was found to be less accurate at very shallow depths for treatment purposes [26], i.e., an uncertainty of pre-treatment depth assessment for shallow tumors was seen. Although, in this study we showed the possibility of using a similar $\mathrm{MHz}$ US in follow-up after completion of treatment and its feasibility in detecting residual disease, the concern for shallow residual disease detection by $18 \mathrm{MHz}$ US persists. Biopsy remains the gold standard for diagnosis of a skin cancer, although various molecular imaging techniques have been studied for skin imaging, including computed tomography, positron emission tomography, and high frequency US [27]. The potential for $30 \mathrm{MHz}$ US to show details to characterize skin carcinoma versus precancerous lesions and healthy skin, has been investigated [28]. This higher frequency US may potentially show important tissue details to support a follow-up. However, this higher frequency US is more experimental, while an $18 \mathrm{MHz}$ US is available in most clinical departments, and as such, more applicable for the feasibility of US use in follow-up.

This study shows the possible utility of US in the follow-up of eBT-treated NMSCs. Eleven patients were shown to have a radiologic CR. Therefore, the 3 patients without a CR on follow-up US were monitored more closely with frequent follow-up by radiation oncologists and dermatologists for possible recurrences. None of our patients had a clinical recurrence requiring salvage treatment with a median follow-up of 20.5 months. However, studies have shown that the majority of recurrences occur in the first 3 years after treatment [16].

Some limitations of this study include a small sample size and a short follow-up, so it may not have been possible to capture probable recurrences that could still occur with further follow-up. A previously published study found significantly different measurements for prescription depth between US and clinical exam in the pre-treatment setting [29]. Our 3 patients with PR were followed with clinical exam and did not have a repeated post-treatment US showing no evidence of disease. Therefore, physicians may not have visualized or palpated PR if there was a subtle residual disease on clinical exam. However, our outcomes and toxicities were similar to 2 year results for BCC eBT [30]. Also, the use of US imaging is operator dependent. Nevertheless, we were able to demonstrate a possible feasibility and potential usefulness of US in the follow-up of NMSCs post-treatment by capturing 3 patients, who continued to have residual disease after eBT that was not detected on clinical examination. Due to the post-treatment US, we have adjusted the clinical follow-up schedule to be more frequent and rigorous in the treated area for patients who had a PR after eBT.

Therefore, we conclude that follow-up US is feasible to evaluate for NMSCs persistence of disease and treatment response after eBT. However, larger studies are needed to determine which patients may benefit the most from follow-up US after eBT. Currently, guidelines suggest clinical physical examination for NMSC follow-up $[2,14]$, but this study shows that US may be an objective follow-up modality to guide further NMSC management.

\section{Conclusions}

US was found to be a feasible imaging modality for post-treatment follow-up after eBT. Follow-up US may allow clinicians to objectively evaluate treatment response, help to determine timing for further follow-up, and possibly, evaluate patients with early recurrences suitable for further salvage treatments. Other studies with a greater number of patients and longer follow-up are needed to confirm and validate the results of our study, and to better establish the follow-up plan using US in NMSC.

\section{Disclosure}

Authors report no conflict of interest.

\section{References}

1. ACS. ACS Cancer Facts 2015. http://uacc.arizona.edu/ sites/default/files/acs_2015_0.pdf. Accessed on September 26, 2017.

2. Newlands C, Currie R, Memon A et al. Non-melanoma skin cancer: United Kingdom National Multidisciplinary Guidelines. J Laryngol Otol 2016; 130 (Suppl 2): S125-S132.

3. NCCN guidelines. Basal cell skin cancer guidelines. https:// www.nccn.org/professionals/physician_gls/pdf/nmsc.pdf. Accessed on October 3, 2016. 
4. NCCN guidelines. Squamous cell skin cancer guidelines. https://www.nccn.org/professionals/physician_gls/pdf/ squamous.pdf. Accessed on October 3, 2016.

5. Paravati AJ, Hawkins PG, Martin AN et al. Clinical and cosmetic outcomes in patients treated with high-dose-rate electronic brachytherapy for nonmelanoma skin cancer. Pract Radiat Oncol 2015; 5: e659-64.

6. Jennings L, Schmults $C D$. Management of high-risk cutaneous squamous cell carcinoma. J Clin Aesthet Dermatol 2010; 3: $39-48$

7. Delishaj D, Manfredi B, Laliscia C et al. Non-melanoma skin cancer treated with HDR Brachytherapy and Valencia applicator in elderly patients. Eur J Cancer 2015; 51: S669.

8. Delishaj D, Rembielak A, Manfredi B et al. Non-melanoma skin cancer treated with high-dose-rate brachytherapy: a review of literature. J Contemp Brachytherapy 2016; 8: 533-540.

9. Gauden R, Pracy M, Avery AM et al. HDR brachytherapy for superficial non-melanoma skin cancers. J Med Imaging Radiat Oncol 2013; 57: 212-217.

10. Tormo A, Celada F, Rodriguez $\mathrm{S}$ et al. Non-melanoma skin cancer treated with HDR Valencia applicator: clinical outcomes. J Contemp Brachytherapy 2014; 6: 167-172.

11. Delishaj D, Laliscia C, Manfredi B et al. Non-melanoma skin cancer treated with high-dose-rate brachytherapy and Valencia applicator in elderly patients: a retrospective case series. J Contemp Brachytherapy 2015; 7: 437-444.

12. Ballester-Sánchez R, Pons-Llanas O, Candela-Juan C et al. Electronic brachytherapy for superficial and nodular basal cell carcinoma: a report of two prospective pilot trials using different doses. J Contemp Brachytherapy 2016; 8: 48-55.

13. Haseltine JM, Parker M, Wernicke AG et al. Clinical comparison of brachytherapy versus hypofractionated external beam radiation versus standard fractionation external beam radiation for non-melanomatous skin cancers. J Contemp Brachytherapy 2016; 8: 191-196.

14. Ouhib Z, Kasper M, Calatayud JP et al. Aspects of dosimetry and clinical practice of skin brachytherapy: The American Brachytherapy Society working group report. Brachytherapy 2015; 14: 840-858.

15. Heskin L, Filobbos G, Heskin JK et al. The compliance of Irish Plastic Surgeons to the guidelines for the management of "non-melanoma skin cancers" (NMSC). Are the follow-up recommendations clear enough? J Plast Reconstr Aesthet Surg 2010; 63: 2192-2193.

16. Zagrodnik B, Kempf W, Seifert B et al. Superficial radiotherapy for patients with basal cell carcinoma: recurrence rates, histologic subtypes, and expression of p53 and Bcl-2. Cancer 2003; 98: 2708-2714.

17. Schmults CD, Karia PS, Carter JB et al. Factors predictive of recurrence and death from cutaneous squamous cell carcinoma: a 10-year, single-institution cohort study. JAMA Dermatol 2013; 149: 541-547.

18. Goyal U, Kim Y, Tiwari HA et al. A pilot study of ultrasound-guided electronic brachytherapy for skin cancer. I Contemp Brachytherapy 2015; 7: 374-380.

19. Pasquali P, Camacho E, Fortuny A. Use of $22 \mathrm{MHz}$ High-Frequency Ultrasound in the Management of Skin Cancer. In: Baldi A (ed.). Skin Cancer, Current Clinical Pathology. Springer Science + Business Media, New York 2014; pp. 245-256.

20. Bauman JE, Eaton KD, Martins RG. Treatment of recurrent squamous cell carcinoma of the skin with cetuximab. Arch Dermatol 2007; 143: 889-892.

21. Matthiesen C, Thompson JS, Forest $\mathrm{C}$ et al. The role of radiotherapy for T4 non-melanoma skin carcinoma. J Med Imaging Radiat Oncol 2011; 55:407-416.

22. Brotherston D, Poon I. SBRT Treatment of Multiple Recurrent Auricular Squamous Cell Carcinomas Following Surgi- cal and Conventional Radiation Treatment Failure. Cureus 2015; 7: e325.

23. Anasagasti-Angulo L, Garcia-Vega Y, Barcelona-Perez S et al Treatment of advanced, recurrent, resistant to previous treatments basal and squamous cell skin carcinomas with a synergistic formulation of interferons. Open, prospective study. BMC Cancer 2009; 9: 262.

24. Czarnecki D, Staples M, Mar A et al. Recurrent nonmelanoma skin cancer in southern Australia. Int J Dermatol 1996; 35: 410-412.

25. Signorell J, Hunziker T, Martinelli M et al. Recurrent nonmelanoma skin cancer: remission of field cancerization after conversion from calcineurin inhibitor- to proliferation signal inhibitor-based immunosuppression in a cardiac transplant recipient. Transplant Proc 2010; 42: 3871-3875.

26. Ballester-Sánchez R, Pons-Llanas $\mathrm{O}$, Llavador-Ros $\mathrm{M}$ et al. Depth determination of skin cancers treated with superficial brachytherapy: ultrasound vs. histopathology. J Contemp Brachytherapy 2015; 6: 356-361.

27. Hong H, Sun J, Cai W. Anatomical and molecular imaging of skin cancer. Clin Cosmet Investig Dermatol 2008; 1: 1-17.

28. Piotrzkowska-Wroblewska H, Litniewski J, Szymanska E et al. Quantitative Sonography of Basal Cell Carcinoma. Ultrasound Med Biol 2015; 41: 748-759.

29. Goyal U, Pan J, Cui H et al. Does ultrasound measurement improve the accuracy of electronic brachytherapy in the treatment of superficial non-melanomatous skin cancer? J Contemp Brachytherapy 2017; 9:14-19.

30. Ballester-Sánchez R, Pons-Llanas O, Candela-Juan C et al. Two year results of electronic brachytherapy for basal cell carcinoma. J Contemp Brachytherapy 2017; 9: 251-255. 